\title{
Galaxy Preprocessing in a Starbrusting Group Infalling into Abell1367
}

\author{
L. Cortese ${ }^{1}$, G. Gavazzi ${ }^{2}$, A. Boselli ${ }^{3}$, P. Franzetti ${ }^{4}$, R. C. Kennicutt ${ }^{5}$, \\ K. O'Neil ${ }^{6}$, and S. Sakai ${ }^{7}$ \\ ${ }^{1}$ School of Physics and Astronomy, Cardiff University, Cardiff CF24 3AA, UK \\ ${ }^{2}$ Universitá degli Studi di Milano-Bicocca, P.zza della Scienza 3, 20126 Milano, Italy \\ ${ }^{3}$ Laboratoire d'Astrophysique de Marseille, Traverse du Siphon, Marseille, France \\ ${ }^{4}$ IASF-INAF, Sezione di Milano, Via E. Bassini 15, I-20133 Milano, Italy \\ ${ }^{5}$ Institute of Astronomy, University of Cambridge, Madingley Road, Cambridge. CB3 0HA,UK \\ ${ }^{6}$ NRAO, P.O. Box 2, Green Bank, WV 24944 \\ ${ }^{7}$ Department of Astronomy, University of California, Los Angeles, CA, 90095-1562
}

\begin{abstract}
We present a multiwavelength analysis of a compact group infalling at high speed $\left(\sim 1700 \mathrm{~km} \mathrm{~s}^{-1}\right)$ into the dynamically young cluster Abell 1367. Peculiar morphologies and unusually high Halpha emission are associated with two giant galaxies and at least ten dwarfs/ extragalactic HII regions, making this group the region with the highest density of star formation activity ever observed in the local clusters. Moreover Halpha imaging observations reveal extraordinary complex trails of ionized gas behind the galaxies, with projected lengths exceeding $100 \mathrm{kpc}$. These unique cometary trails mark the gaseous trajectory of galaxies, witnessing their dive into the hot cluster intergalactic medium. Under the combined action of tidal forces among group members and the ram-pressure by the cluster ambient medium, the group galaxies were fragmented and the ionized gas was blown out. The properties of this group suggest that environmental effects within infalling groups may have represented a preprocessing step of the galaxy evolution during the high redshift cluster assembly phase.
\end{abstract}

Keywords. galaxies: evolution-galaxies:clusters: individual: Abell1367-galaxies:interactionsgalaxies: intergalactic medium
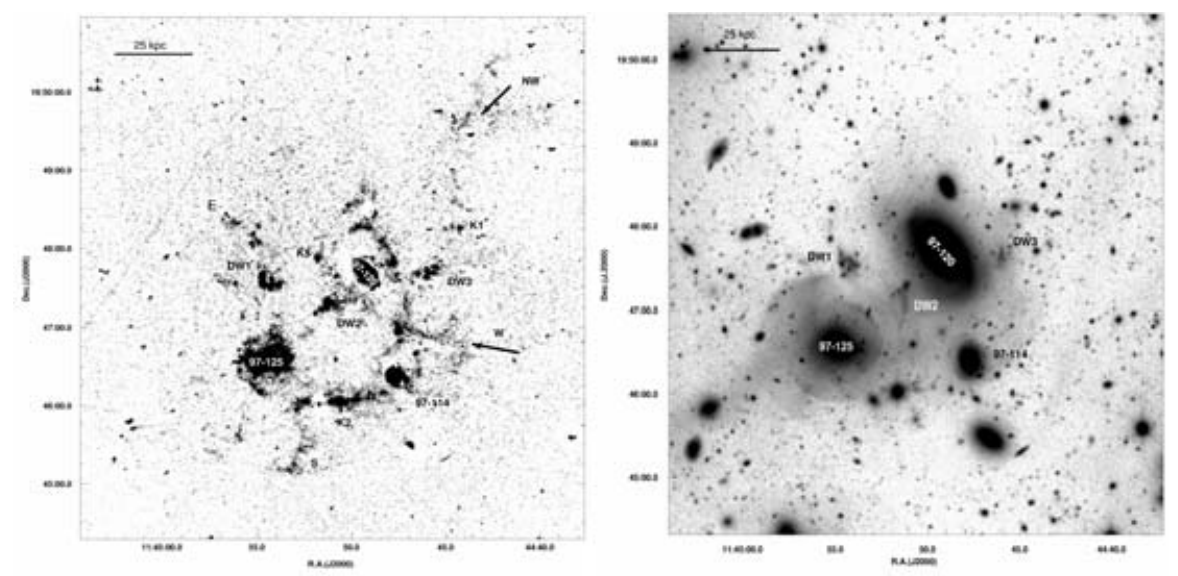

Figure 1. Left: Extended low brightness loops and trails (labeled NW, W and E) appear in the $\mathrm{H} \alpha+[\mathrm{NII}]$ NET frame around the giant and dwarf galaxies in the infalling group. Right: Stellar shells are seen around galaxy CGCG97-125 in the $r^{\prime}$ band image of the infalling group. 